J. Phys. IV France 1 (2018)

$\operatorname{Pr} 1-1$

(c) EDP Sciences, Les Ulis

\title{
Fate of the Wigner crystal on the square lattice
}

\author{
D. Baeriswyl ${ }^{1}$ and S. Fratini ${ }^{2}$ \\ ${ }^{1}$ Department of Physics, University of Fribourg, Chemin du musée 3, CH-1700 Fribourg \\ ${ }^{2}$ LEPES-CNRS, 25 avenue des Martyrs, F-38042 Grenoble Cedex 9
}

\begin{abstract}
The phase diagram of a system of electrons hopping on a square lattice and interacting through long-range Coulomb forces is studied as a function of density and interaction strength. The presence of a lattice strongly enhances the stability of the Wigner crystal phase as compared to the case of the two-dimensional electron gas.
\end{abstract}

\section{INTRODUCTION}

At very low densities the ground state of an ensemble of charged particles is a Wigner crystal, ' with a triangular structure if the motion is restricted to two dimensions. This striking phe' nomenon has been observed for a very dilute electron system on the surface of liquid helium. In solids the already existing lattice periodicity interferes with the generally different ordering tendency of the electrons. We have previously studied this competition between Wigner crystallization and lattice commensurability for spinless fermions hopping between the sites of a one-dimensional lattice and coupled pair-wise by long-range Coulomb forces $[1,2]$. In this case charge ordering is found to occur for any density and interaction strength, and only the amplitude of the modulation changes, from a Wigner superlattice for very large interaction strength to a small amplitude charge-density wave at weak coupling.

Here we extend our considerations to the square lattice where a metal-insulator transition is expected to occur as a function of coupling strength. We first consider the classical limit where the ground state structures show interesting variations as the filling is varied. We then determine a crossover region where the particles begin to spill over to neighbouring sites due to quantum fluctuations. Finally we estimate the critical interaction strength for which the Wigner crystal melts. For small densities the continuum result is expected to remain valid, while lattice commensurability effects become very important at larger densities.

We consider the following Hamiltonian of spinless fermions,

$$
H=-t \sum_{<i, j>}\left(c_{i}^{\dagger} c_{j}+c_{j}^{\dagger} c_{i}\right)+\frac{1}{2} \sum_{i, j} V_{i \neq j}\left(n_{i}-n\right)\left(n_{j}-n\right),
$$

where $V_{i, j}=V / d_{i, j}, V$ being the nearest-neighbour interaction, $n_{i}=c_{i}^{\dagger} c_{i}$ and $d_{i, j}$ is the distance between sites $i$ and $j$ of a square lattice. The effect of a constant compensating charge is explicitly included in the Coulomb term. Our aim is to determine the ground state as a function of both $n$, the average number of particles per site, and the relative interaction strength $V / t$. 


\section{CLASSICAL LIMIT}

The classical problem $(t=0)$ has been first addressed by Pokrovsky and Uimin [3], who determined the ground-state structures for a few rational fillings $n=1 / q, q$ being a small integer. Later Cocho proposed a simple recipe for generating the lowest-energy configurations for rational numbers $n=p / q, p>1$ [4]. In order to extract the effects of lattice commensurability we have calculated numerically the energies for the simple rational fillings $n=1 / q$, assuming a Bravais lattice with one electron per unit cell. The procedure can be directly generalised to other rational fillings.

A perfect triangular structure on a host square lattice can only be realized in the limit $n \rightarrow 0$. Thus a measure of commensurability effects is the difference between the energy of the ground state configuration and that of such an ideal structure, given by

$$
E_{\text {ideal }}=(-1.9605 \sqrt{n}+1.9501 n) V
$$

per particle. Our results are illustrated in Fig. 1 for the series $n=1 / q$. The mismatch between the configuration on the lattice and the ideal structure is typically below $1 \%$ at fillings $n<0.1$, but it clearly does not follow a monotonously increasing trend. Particularly stable ground state configurations appear for example at $n=1 / 8,1 / 12,1 / 15$, which are good approximants to the ideal triangular structure. Other configurations, such as $n=1 / 7$, are less stable because of widely different nearest-neigbour distances or bond angles.

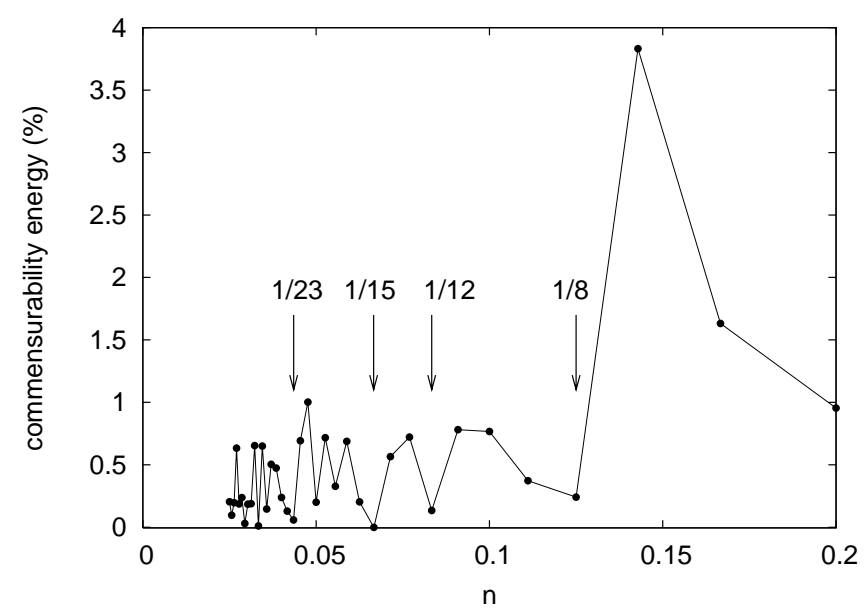

Fig. 1. The deviation of the energy of the ground state configuration from that of a perfect triangular ordering, which gives a measure of the commensurability effects. Arrows mark the ground state structures which are good approximants to the optimal triangular structure.

\section{QUANTUM SPREADING}

A finite hopping term leads to excursions of particles away from their classical equilibrium positions. For small values of $t$ the displacement will be mostly to nearest neighbor sites, creating dipoles of length $a_{0}$, the lattice constant of the host crystal. The energy of an individual dipole can be estimated in the continuum limit, i.e. for an ideal triangular structure. We find

$$
E_{\text {dip }} \approx 0.735 n^{3 / 2} V \text {. }
$$


To lowest order in perturbation theory the density on a nearest neighbor site of a superlattice point is given by

$$
\delta n=\left(t / E_{d i p}\right)^{2} .
$$

For appreciable spreading, say if the probability of finding the particle on one of the four neighboring sites is about one half, we expect the discrete nature of the underlying square lattice to become unimportant. Together with Eqs. (3.1) and (3.2) this gives a crossover region around

$$
V / t \approx 3.85 n^{-3 / 2}
$$

between the parameter ranges of strong and weak discreteness effects.

\section{QUANTUM MELTING}

Our relation for the crossover region, Eq. (3.3), shows that for very small densities, discreteness effects are important only above an extremely large interaction strength (or an extremely small hopping parameter). Below this value the physics of our model is expected to be equivalent to that of the "two-dimensional electron gas", for which extensive numerical simulations have been performed. It is widely accepted that this system undergoes a melting transition from a triangular Wigner crystal to a correlated liquid at $r_{s} \approx 37$ [5]. In our model the corresponding parameter is obtained by identifying the tight-binding spectrum for $k \rightarrow 0$ with a parabolic spectrum (with $\hbar^{2} /\left(2 m^{*} a_{0}^{2}\right)=t$ ) and by introducing an effective charge through the relation $\left(e^{*}\right)^{2} / a_{0}=V$. Thus the effective Bohr radius is given by $a_{B}^{*}=2 a_{0} t / V$. Together with the relation $\pi\left(r_{s} a_{B}^{*}\right)^{2}=a_{0}^{2} / n$ for the surface per particle we obtain the following estimate for the melting transition at very small densities

$$
V / t \approx 131 n^{1 / 2}
$$

Fig. 2 shows that for fillings above about 0.2 this line enters the region where discreteness effects become important. For these fillings Eq. (4.1) can no longer be trusted and we have to go back to the original model, Eq. (1.1). A simple way for estimating the melting line consists in using variational wave functions, one for the Wigner crystal, the other for the metallic state. The most obvious choice is the ground state of the Coulomb term for the insulating phase (corresponding to the classical Wigner superlattice) compared to the ground state of the hopping term for the metallic phase (the restricted Hartree-Fock approximation). The energy of the classical (ideal) Wigner crystal is given by

$$
E_{W C}=\left(-1.96 n^{1 / 2}+1.95 n\right) V,
$$

while the Hartree-Fock solution can be expanded as

$$
E_{H F}=\left(-1.51 n^{1 / 2}+1.95 n\right) V-(4-2 \pi n) t+\mathcal{O}\left(n^{3 / 2}\right) .
$$

As expected, the former has lower energy for large $V / t$, while the latter wins for small values of $V / t$. The energies are equal for

$$
V / t \approx 8.9 n^{-1 / 2}-14.0 n^{1 / 2} .
$$

This result (dotted line in Fig. 2) reproduces rather well the numerical values (dots in the figure), which are based on the actual energies of the different classical configurations and the (numerical) Hartree-Fock energies for the different densities. 


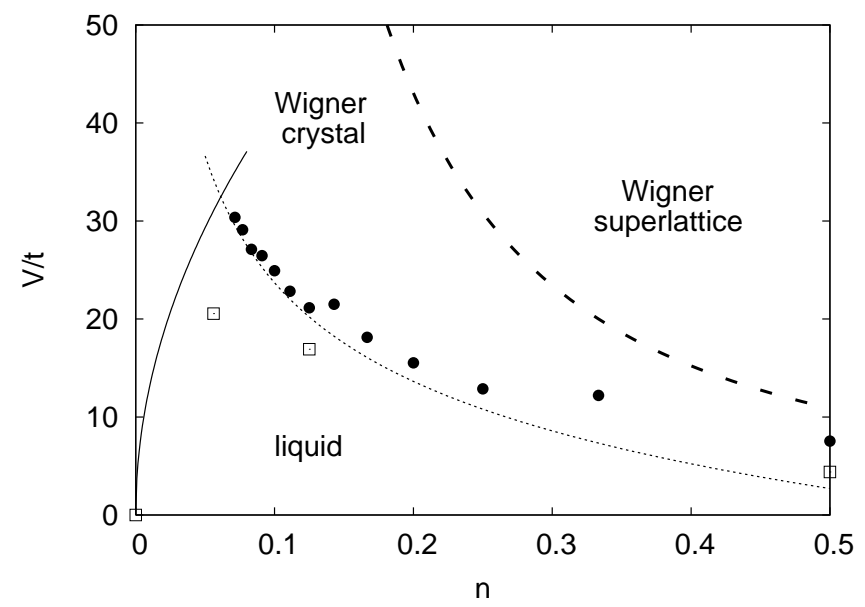

Fig. 2. Phase diagram. The full line corresponds to the continuum limit [5], the dotted line to Eq. (4.4). The dots represent our numerical results, the empty squares those of Ref. [6].

Our numerical results are expected to be quite reliable for densities close to $n=0.5$ where they are not far away from the crossover line. However, for lower densities we expect that correlation effects will stabilize the liquid phase [6]. Therefore we anticipate that below $n \approx 0.3$ the true melting line would be above our numerical results and then smoothly join that of the electron gas. At first sight this seems to contradict the results of Noda and Imada [6], reproduced in Fig. 2 as empty squares, but one has to keep in mind that by fine-tuning the underlying lattice they have stabilized artificially the Wigner crystal phase.

\section{DISCUSSION}

We have studied the stability of the Wigner crystal phase on a square lattice. The phase diagram (interaction strength $V / t$ versus density $n$ ) shows different regimes. For very small densities $(n<0.1)$ the melting line is that of the two-dimensional electron gas. Strong deviations occur close to $n=0.5$ where the Wigner crystal is stabilized by the lattice (the motion is more and more hindered as the density increases). In-between, the melting line is expected to go through a maximum. Despite the stabilizing effects of the lattice the Wigner crystal is still only stable for very large interaction strengths $\left(V_{c, \min } \approx 6 t\right)$. Therefore Coulomb interactions alone are unlikely to promote charge ordering in layered cuprates where $V \approx t$. In these and related materials other effects, in particular three-dimensionality [7], electron-lattice coupling, counter-ions or magnetic exchange may be necessary to stabilize charge-ordered structures.

\section{References}

[1] B. Valenzuela, S. Fratini and D. Baeriswyl, Phys. Rev. B 68, 045112 (2003).

[2] S. Fratini, B. Valenzuela and D. Baeriswyl, Synth. Met. 141, 193 (2004).

[3] V. L. Pokrovsky and G. V. Uimin, J. Phys. C 11, 3535 (1978).

[4] G. Cocho, R. Perezpascual and J. L. Rius, Europhys. Lett. 2, 493 (1986).

[5] B. Tanatar and D. M. Ceperley, Phys. Rev. B 39, 5005 (1989).

[6] Y. Noda and M. Imada, Phys. Rev. Lett. 89, 176803 (2002).

[7] G. Rastelli and S. Fratini, this issue 